\title{
Security Evaluation System for Android Applications Using User's Reviews and Permissions
}

\author{
Naonobu Okazaki \\ University of Miyazaki \\ 1-1 Gakuen-Kibanadai-Nishi, Miyazaki, 889-2192, Japan \\ Yoshihiro Kita \\ Tokyo University of Technology \\ 1404-1 Katakura, Hachioji, 192-0982, Japan \\ Kentaro Aburada \\ Oita National College of Technology \\ 1666 Ooaza-Maki, Oita, 870-0152, Japan \\ Mirang Park \\ Kanagawa Institute of Technology \\ 1030 Shimo-Ogino, Atsugi, 243-0292, Japan
}

\begin{abstract}
Leakage of the important data in mobile terminals by mal-applications is becoming a serious threat. The users must be careful not to install mal-applications. Application markets provide the application's reviews and using permissions at the application downloading. However, the most of users check the reviews only. All users must be cautious about not only the using permissions but also the combination of them. In this paper, we propose a security evaluation system to prevent the installation of mal-applications on Android OS. This system indicates the user reviews with the using permission information of application to new users.
\end{abstract}

Keywords: Mal-applications, User's reviews, Permissions, Android OS.

\section{Introduction}

Recently, people have stored important data and some applications in mobile terminals, e.g. smartphones and tablet PC, with the spread of them. Leakage of important data, e.g. personal information and confidential information, in the mobile terminals by mal-applications is becoming a serious threat.

On the market side, Google implements the malapplication detection system "Bouncer" for Google play[1] to take countermeasure of the matter. On the developer side, application development and management system "ADMS"[2] is the system which uses the security manager, analyzes the application to prevent mal-application from spreading to market at releasing. However, mal-applications are not eliminated completely. It is important to be careful not to install mal-application on the user side.

The application markets, e.g. Google play, provide the application's reviews and using permissions to new users at the downloading of an application. However, the most of new users check the reviews only, because it is difficult for them to understand the using permissions. Some of existing reviews are useless because they are malicious or the unrelated to contents of the application. So, the mal-application detection is hard to new user from the reviews and using permissions. 
In this paper, we propose a security evaluation system using user's reviews and permissions for Android OS. This system shows the existing user's reviews and using permissions of application to new users. User's reviews are assessed to new users at the downloading of the application. Our system transmits the assessment of review to the reviewer, and weeds out the reviews which are selected as malicious or useless by new users. The reviewer refers to the assessment of review and contributes to improvement of a review.

\section{Related Works}

Reviews are useful as guidelines for new users to download the application. However, if the malapplication developer writes the review of the application in order to be installed it by users, this review is exaggerated review or fake review. The other users trust these review, and are suffered damage by mal-applications.

It is impossible to remove the vulnerability of applications completely, but guidelines of secure applications for Android developers (i.e. iSEC Partner[3]) are issued by various organizations for security information.

Existing review system (i.e. Google play[1]) show reviews of user and using permissions to new users. It is possible to predict the risk of application from reviews and permissions, because these are indicate the behavior of application. But, users can not understand the risk of application adequately, because these are not indicate the risk of application in combination with other permissions, and it is difficult to understand the behavior of application by users who do not understand the expertise of security and function of mobile terminals.

Matsudo[4] proposed security advisor system, which indicates the risk of application by the combination of permissions. This system shows the number of download and the risk level of application to new users. However, these values are not objective and fair measure, because other users can manipulate these values intentionally, and new users do not have information enough to determine the application downloading by these values only. It needs for new users to show the user's reviews of applications in order to get the information of applications.

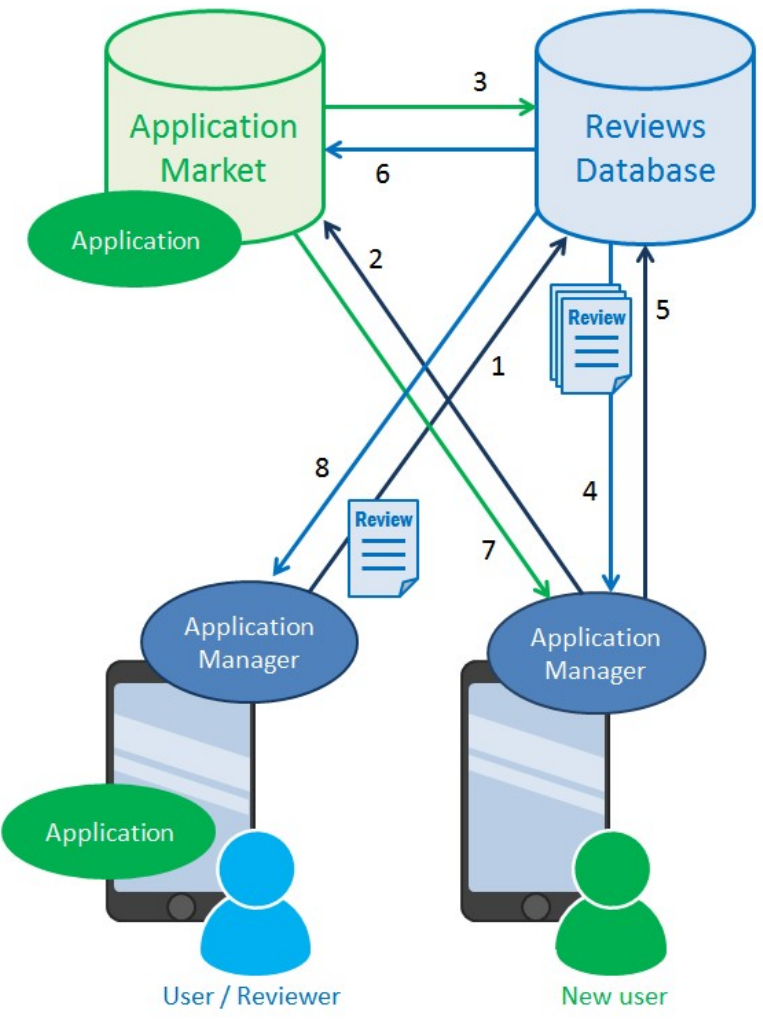

Fig. 1. Structure of security evaluation system.

\section{Security Evaluation System}

We propose security evaluation system, which indicate the risk of application by the reviews and using permissions. This system indicates the reviews and the assessment of them. Fig.1 shows the structure of security evaluation system. This system consists of two parts as follows:

\section{- Reviews Database}

This is storage of reviews and the assessment of reviews, which are sent from all users.

- Application Manager

This is the application which indicates the reviews and the risk of applications by the combination of permissions to terminal user, and transmits the user's review to review database.

The procedure of this system is described as follows: 
Table 1. Risk allowances of applications.

\begin{tabular}{|c|c|c|c|}
\hline & Safety & Caution & Danger \\
\hline $\begin{array}{l}\text { Does the application have the } \\
\text { one or more dangerous } \\
\text { permission? }\end{array}$ & $\mathrm{NO}$ & YES & YES \\
\hline $\begin{array}{l}\text { Does the application have the } \\
\text { dangerous combination of } \\
\text { permissions? }\end{array}$ & NO & $\mathrm{NO}$ & YES \\
\hline $\begin{array}{l}\text { Is the application reported as } \\
\text { mal-application? }\end{array}$ & NO & $\mathrm{NO}$ & YES/NO \\
\hline
\end{tabular}

1. The user, who is using the application, sends a review to review database through the user's application manager.

2. New user requests the download of the application to the application market.

3. The application market sends the information of the application (i.e. application's name, developer, request user's name) to reviews database.

4. Reviews database shows the all review and the assessment of them for the application to new user through the new user's application manager.

5. New user gives the assessment "Good" or "Bad" for the one of the reviews, and reports the assessment of the review to reviews database through the application manager.

6. Reviews database stores the assessment of review, and permit the download of the application to application market.

7. New user downloads the application to application market through the application manager.

8. Reviews database reports the assessment of the review to existing user as reviewer.

Reviews have two types: positive reviews and negative reviews. Positive reviews include selling points or good features for the application. Negative reviews include wrong points or problems of the application. The reviews are evaluated not only new users but also existing user instead of writing the review.

The permissions have the four protection levels[5]: "normal," "dangerous," "signature," and "signatureOfSystem." Table.1 shows the risk allowances of applications that we defined. We divide the risk of applications into three levels as follows
Table 2. The permissions concern the personal information or the information leak.

\begin{tabular}{ll}
\hline $\begin{array}{l}\text { Permissions concern personal } \\
\text { information }\end{array}$ & $\begin{array}{l}\text { Permissions concern } \\
\text { information leak }\end{array}$ \\
\hline READ_CONTACTS & INTERNET \\
WRITE_CONTACTS & SEND_SMS \\
READ_CALENDAR & BLUETOOTH \\
WRITE_CALENDAR & NFC \\
READ_LOGS & USE_SIP \\
BIND_APPWIDGET & CHANGE_NETWORK_STATE \\
READ_PROFILE & BLUETOOTH_ADMIN \\
WRITE_PROFILE & CALL_PHONE \\
ACCESS_FINE_LOCATION & \\
ACCESS_COARSE_LOCATION & \\
ACCESS_MOCK_LOCATION & \\
GET_ACCOUNT & \\
READ_EXTRNAL_STORAGE & \\
WRITE_EXTRNAL_STORAGE & \\
READ_HISTORY_BOOKMARKS & \\
WRITE_HISTORY_BOOKMARKS & \\
DUMP & \\
AUTHENTICATE_ACCOUNTS & \\
READ_PHONE_STATE & \\
READ_INPUT_STATE & \\
ASEC_ACCESS & \\
GET_TASKS &
\end{tabular}

according to the protection levels and the combination of permissions.

- Safety

All permissions use the protection level "normal" only.

- Caution

Permissions use the protection level "dangerous,"

"signature," or "signatureOfSystem."

- Danger

The application is permitted the functions which include both connecting internets and accessing the personal information, plus the condition of "Caution."

Table. 2 shows the permissions concern the personal information or the information leak. The applications include these permissions are allocated the risk of applications "Danger."

Fig.2 shows the example of indication for risk allowances of applications. The application manager indicates the risk of the application using the kinds of three colors for each risk and descriptions of the using 


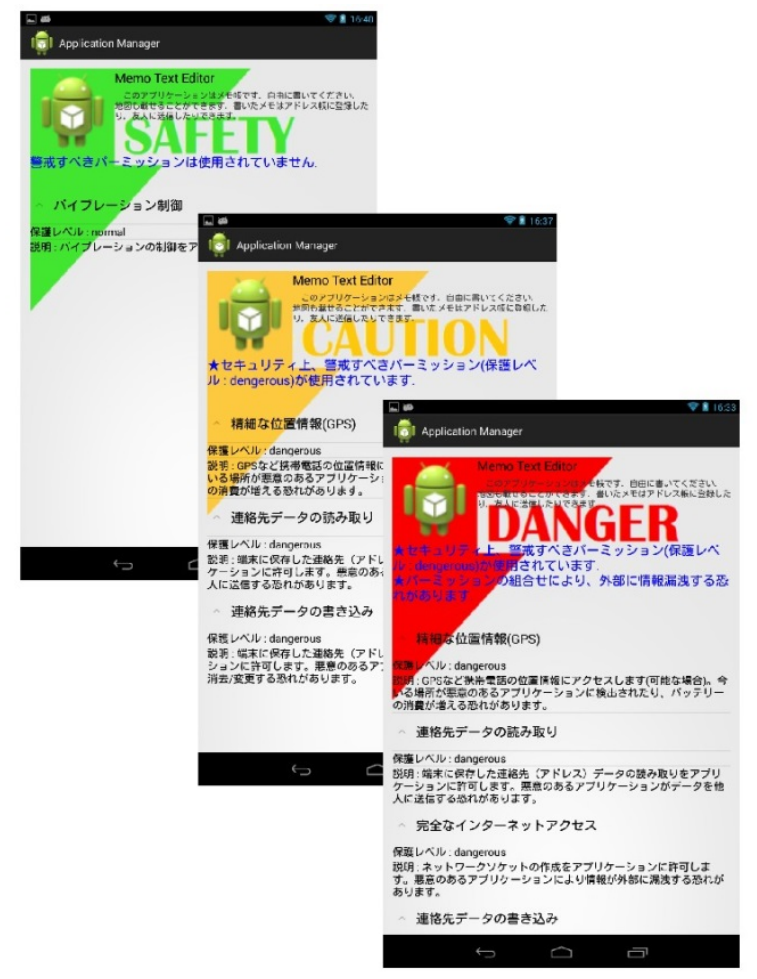

Fig. 2. Example of indication for risk allowances of applications.

permissions. The new users can recognize the dangerous applications easily by these indications.

\section{Consideration}

New users can understand the behavior of applications in detail by the reviews. It is important for all users to understand the risk of application correctly. It needs to show the reviews and the risk of combination of using permissions for new users to determine the application downloading. Table. 3 shows the indication of each method. Google play does not indicate the risk of combination of using permissions. Security advisory system[4] does not show the reviews of users. These methods are insufficient to prevent the installation of mal-applications. Security evaluation system indicates the reviews and the risk on combination of using permission. Therefore, security evaluation system is useful method for new user to prevent installation of the mal-application.

However, all of the dangerous applications are not the mal-applications. It is difficult for users to recognize the mal-applications from all of the dangerous applications
Table 3. Indication by each method.

\begin{tabular}{llll}
\hline & Google play[1] & $\begin{array}{l}\text { Security } \\
\text { advisory } \\
\text { system[4] }\end{array}$ & $\begin{array}{l}\text { Security } \\
\text { evaluation } \\
\text { system }\end{array}$ \\
\hline $\begin{array}{l}\text { Risk of permissions } \\
\text { Risk of combination of } \\
\text { using permissions } \\
\text { Reviews }\end{array}$ & $\begin{array}{l}\text { Indicate } \\
\text { No Indicate }\end{array}$ & $\begin{array}{l}\text { Indicate } \\
\text { Indicate }\end{array}$ & $\begin{array}{l}\text { Indicate } \\
\text { Indicate }\end{array}$ \\
& Indicate & No Indicate & Indicate
\end{tabular}

even if they use the security evaluation system. It needs to improve security evaluation system to recognize the mal-application more exactly.

\section{Conclusion}

We proposed a security evaluation system using user's reviews and permissions for Android OS in order to prevent installation of the mal-application. This system indicates the reviews, the assessment of review, and the risk of combination of using permissions. The reviewer refers the assessment of the reviewer's review, and will be written the useful review next time. Therefore, new users can recognize the mal-applications by useful reviews and the indication of risk for installing the applications.

\section{References}

[1] "Google play," Google. http://play.google.com/store

[2] H. Agematsu, J. Kani, K. Nasaka, H. Kawabata, T. Isohara, K. Takemori, and M. Nishigaki, "A Proposal to Realize the Provision of Secure Android Application ADMS: An Application Development and Management System," in Proc. 6th Int. Conf. Innovative Mobile and Internet Services in Ubiquitous Computing (IMIS), pp.677-682 (2012).

[3] J. Burns, "Developing Secure Mobile Application for Android," iSEC Partners (2008).

[4] T. Matsudo, E. Kodama, J. Wang, and T. Takata, "A Proposal of Security Advisory System at the Time of the Installation of Applications on Android OS," in Proc. 15th Int. Conf. Network-Based Information Systems (NBiS), pp.261-267 (2012)

[5] B. M. A. Egner and U. Meyer, "Messing with Android's Model," in Proc. the 2012 IEEE 11th Int. Conf. Trust, Security and Privacy in Computing and Communications, pp.504-514 (2012) 